\title{
Kinetic Phase Transition in the Mixed-Spin Ising Model
}

\author{
M. Godoy and W. Figueiredo \\ Departamento de Física, Universidade Federal de Santa Catarina, 88040-900, Florianópolis, SC, Brazil
}

Received on 9 August, 2003

\begin{abstract}
In this work we studied a ferromagnetic mixed-spin Ising model including a single ion crystal-field term. The model system consists of two interpenetrating sublattices with spins $\sigma=1 / 2$ and $S=1$. The spins $\sigma=1 / 2$ occupy the sites of one sublattice, their nearest-neighbours are spins $S$ on the other sublattice, and vice versa. The system is in contact with a heat bath, the spins flipping according to the Metropolis transition rate and, at the same time, subject to an external flow of energy, which is simulated by a two-spin flip process. The model is studied via the dynamical pair approximation and through Monte Carlo simulations. We have determined the phase diagram of the model in the plane crystal-field $D$ versus competition parameter $p$. The parameter $p$ accounts for the competition between the one- and two-spin flip processes. In the pair approximation, the phase diagram, at high temperatures, present three phases separated by two transition lines: a continuous transition line between the ferromagnetic and paramagnetic phases, and a first-order transition line between the paramagnetic and antiferromagnetic phases. However, Monte Carlo simulations predict the same topology for the phase diagram as the pair approximation, but all the transition lines are continuous for any value of the temperature.
\end{abstract}

The mixed-spin Ising model has received much attention in the last years. The main motivation is that the model, containing spins of different magnitudes, is used to understand the behavior of certain ferrimagnetic systems that are of great technological interest. From a theoretical point of view many different approaches have been employed in the study of this model: effective-field theories with correlations $[1,2]$, the mean-field renormalization group [3, 4], the Bethe-Peierls method [5], and Monte Carlo simulations $[7,6]$. In these studies, just the equilibrium states were investigated.

In the study of the stationary states of the nonequilibrium thermodynamic systems we do not have at our disposal a closed formalism to deal with these systems as in the equilibrium problems $[8,9]$. In this work we shall consider a mixed-spin Ising model defined on a square lattice, with spins $\sigma=1 / 2$ and $S=1$. The lattice is divided into two interpenetrating sublattices, with the $\sigma$ spins occupying the sites of one sublattice, while the $S$ spins occupy the sites of the other sublattice, each sublattice containing $N$ sites. A state of the system is represented by $(\sigma, S) \equiv\left(\sigma_{1}, \ldots, \sigma_{j}, \ldots, \sigma_{N} ; S_{1}, \ldots, S_{i}, \ldots, S_{N}\right)$, where the spin variables $\sigma_{j}$ can assume the values \pm 1 , and the spin variables $S_{i}$ assume values \pm 1 and 0 . The system is represented by the following hamiltonian model

$$
\mathcal{H}=-J \sum_{\langle i, j\rangle} S_{i} \sigma_{j}-D \sum_{i} S_{i}^{2},
$$

where the sum is over all nearest neighboring pairs of spins. $J$ is the coupling constant between nearest neighbors spins and $D$ is the crystal-field contribution.

Let us call $p(\sigma, S ; t)$ the probability of finding the system in the state $(\sigma, S)$ at time $t$. The equations of motion for the probability of the states of the system is given by the master equation

$$
\begin{aligned}
& \frac{d}{d t} p(\sigma, S ; t)=-\sum_{\sigma^{\prime}, S^{\prime}} W\left(\sigma, S \rightarrow \sigma^{\prime}, S^{\prime}\right) p(\sigma, S ; t) \\
& +\sum_{\sigma^{\prime}, S^{\prime}} W\left(\sigma^{\prime}, S^{\prime} \rightarrow \sigma, S\right) p\left(\sigma^{\prime}, S^{\prime} ; t\right),
\end{aligned}
$$

where $W\left(\sigma, S \rightarrow \sigma^{\prime}, S^{\prime}\right)$ is the probability, per unit of time, for the transition from the state $(\sigma, S)$ to the state $\left(\sigma^{\prime}, S^{\prime}\right)$. In this model, we assumed that the transition rate $W\left(\sigma, S \rightarrow \sigma^{\prime}, S^{\prime}\right)$ is due to the competition between two independent stochastic processes. First, the one-spin flip Glauber process [10], intended to describe the relaxation of the spins $\sigma$ and $S$ in contact with the heat bath. This transition rate can be written as

$$
\begin{aligned}
W_{G}\left(\sigma, S \rightarrow \sigma^{\prime}, S^{\prime}\right)= & W_{G}\left(\sigma, S \rightarrow \sigma^{\prime}, S\right) \\
& +W_{G}\left(\sigma, S \rightarrow \sigma, S^{\prime}\right) .
\end{aligned}
$$

Second, the two-spin flip process, chosen to be independent of temperature, and meant to increase the energy of the system, can be written as $W_{G D}\left(\sigma, S \rightarrow \sigma^{\prime}, S^{\prime}\right)$. Then, we have the following equation for the total transition probability:

$$
\begin{aligned}
& W\left(\sigma, S \rightarrow \sigma^{\prime}, S^{\prime}\right)=p W_{G}\left(\sigma, S \rightarrow \sigma^{\prime}, S^{\prime}\right) \\
& +(1-p) W_{G D}\left(\sigma, S \rightarrow \sigma^{\prime}, S^{\prime}\right),
\end{aligned}
$$

where $0 \leq p \leq 1$ is the competition parameter between the one- and two-spin flip processes. From the master equation we found the equations of motion for the sublattice magnetizations $m_{1}=\left\langle\sigma_{j}\right\rangle$ and $m_{2}=\left\langle S_{i}\right\rangle$, and also for the correlation functions $q=\left\langle S_{i}^{2}\right\rangle, q_{1}=\left\langle\sigma_{j} S_{i}^{2}\right\rangle$ and $r=\left\langle\sigma_{j} S_{i}\right\rangle$ as in ref. [11]. 
These mean values are calculated using the following transition rates: for the one-spin flip transition rate, which simulates the contact of the system with the heat bath, we used the Metropolis prescription, given by

$$
\omega_{j}(\sigma)=\min \left[1, \exp \left(-\beta \Delta E_{j}\right)\right]
$$

where $\beta=1 / k_{B} T$, and $T$ is the absolute temperature of the heat bath. $\Delta E_{j}$ is the change in energy after flipping the spin $\sigma_{j}$ at site $j$. We also assumed a similar expression for $\omega_{i}(S)$. On the other hand, for the two-spin flip transition rate, which favors the increase of the energy of the system, we chose the following rule

$$
\omega_{i j}(S, \sigma)=\left\{\begin{array}{llc}
0 & \text { if } \quad \Delta E_{i j} \leq 0 \\
1 & \text { if } \quad \Delta E_{i j}>0
\end{array}\right.
$$

where $\Delta E_{i j}$ is the change in energy after flipping the spins $S_{i}$ and $\sigma_{j}$, at the neighboring sites $i$ and $j$.

In the present study of this model we used the dynamical pair approximation and Monte Carlo simulations. The set of equations of motion obtained from the master equation can be solved through the dynamical pair approximation [11], and the steady states of the system can be found as a function of $T, p$ and $D$. The equations are solved numerically by employing the fourth-order Runge-Kutta method. The solutions and the corresponding phases are defined in the following way: ferromagnetic phase $(\mathrm{F}), m_{1} \neq m_{2}$, $m_{1}$ and $m_{2}$ having the same signs; paramagnetic phase (P), $m_{1}=m_{2}=0$; antiferromagnetic phase (AF), where $m_{1} \neq m_{2}, m_{1}$ and $m_{2}$ having different signs.

We present in Fig. 1, the phase diagram of the model in the plane crystal-field intensity $D$ versus competition parameter $p$, for two selected values of temperature. For these temperatures, the phase diagram displays three different phases separated by different transition lines. For the values of $p$ in the range $1 \geq p \gtrsim 0.96$, and $D>-4.0 \mathrm{~J}$, the phase diagram presents only a ferromagnetic phase (F). At low temperatures, $T=0.1 \mathrm{~J}$ in Fig. 1 , the continuous line changes into a first-order line for negative values of $D$. The transition between the ferromagnetic $(\mathrm{F})$ and paramagnetic (P) phases exhibits a tricritical point. Increasing the temperature beyond $T=0.262 \mathrm{~J}$ the tricritical behavior disappears. The area occupied by the ferromagnetic phase $(F)$ increases when the temperature decreases. On the other hand, for small values of $p$, what corresponds to a large flow of energy into the system, the phase diagram presents the antiferromagnetic $(\mathrm{AF})$ and paramagnetic $(\mathrm{P})$ phases, which are separated by a first-order transition line. For this model, this transition line is almost independent of temperature.

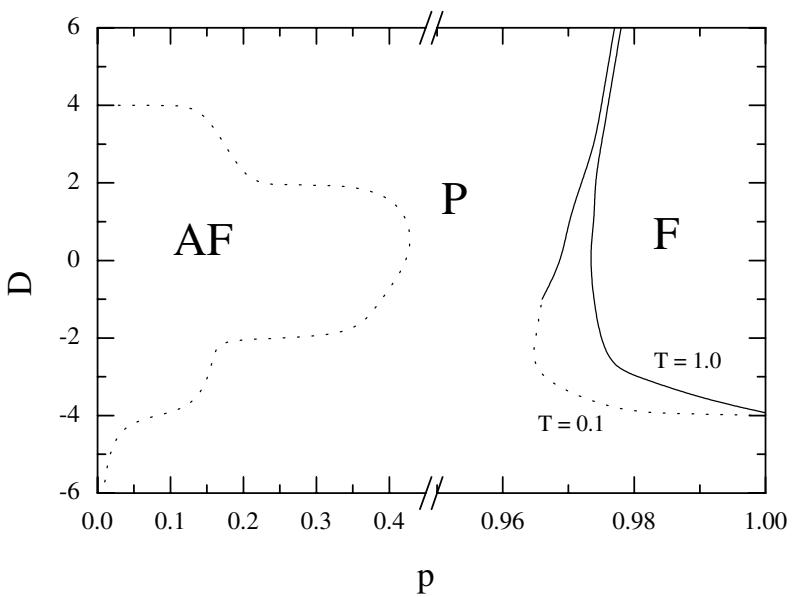

Figure 1. Phase diagram in the plane crystal-field $D$ vs competition parameter $p$, for two selected temperatures, in the dynamic pair approximation. The letters $\mathrm{F}, \mathrm{AF}$ and $\mathrm{P}$, denote ferromagnetic, antiferromagnetic and paramagnetic phases, respectively. The solid lines represent continuous phase transitions, and the dashed lines are the first-order transition points. The temperatures are indicated in the figure.

We also performed Monte Carlo simulations for this model, in order to get a better understanding of the phase transitions found from the pair approximation calculations. We considered a square lattice of linear size $L$, with $L$ ranging from $L=16$ to $L=128$, and we applied periodic boundary conditions. For each simulation run, the initial states of the system were completely random. A new configuration is generated by the following Markov process: for selected values of the temperature $T$, crystal-field intensity $D$, and the competition parameter $p$, we choose a spin at random on the lattice, and we generate a random number $\xi$ between zero and one. If $\xi \leq p$ we considered the one-spin flip process, according to the heat bath algorithm. On the other hand, if $\xi>p$, then we consider the two-spin flip process. In this case, we randomly select another spin, which is a nearest neighbor of the initial spin, and then the state of the system is changed only if energy increases. We discarded the initial $5 \times 10^{4}$ MCS (Monte Carlo steps) until the system enters in a stationary regime. Then, we used more $5 \times 10^{4}$ MCS to calculate the averages of the quantities of interest. One MCS corresponds to $L^{2}$ one- and two-spin flip trials.

We calculated the sublattice magnetization per spin, $m_{1}=\frac{1}{N}\left\langle\sum_{i} S_{i}\right\rangle$ and $m_{2}=\frac{1}{N}\left\langle\sum_{j} \sigma_{j}\right\rangle$. The transition lines of the phase diagram were obtained from the total and staggered magnetizations, defined as $m_{L}^{F}=\frac{1}{2}\left|\left(m_{1}+m_{2}\right)\right|$ and $m_{L}^{A F}=\frac{1}{2}\left|\left(m_{1}-m_{2}\right)\right|$, respectively. We also calculated the reduced fourth-order Binder cumulants associated with these magnetizations. From the crossing point of the cumulants for different lattice sizes we determined the critical points of the model.

We also found the phase diagram of the model in the plane crystal-field $D$ versus competition parameter $p$ from the results of the simulations. Fig. 2 shows the phase diagram of the model for two different values of temperature. As in the pair approximation we obtain the same three dif- 
ferent phases observed in Fig. 1. However, in the Monte Carlo simulations, all the transition lines are continuous, and no tricritical point is observed. For large values of $p$ $(0.95 \leq p \leq 1.0)$ and $D \geq-4 J$, Fig. 2 shows only a ferromagnetic phase $(\mathrm{F})$. The area occupied by this phase also decreases for increasing values of the temperature. On the other hand, the antiferromagnetic phase appears only for very small values of $p$. In Fig. 2 we see that it is restricted to $0.0 \leq p \leq 0.06$ region. Compared with the phase diagram obtained in the context of the pair approximation, we note that the paramagnetic phase $(\mathrm{P})$ occupies a very large area.

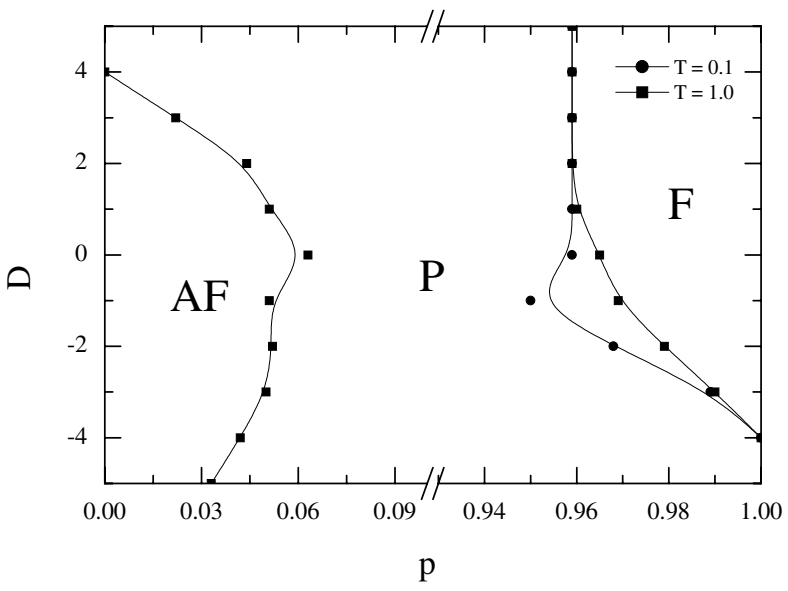

Figure 2. Phase diagram in the plane crystal-field $D$ vs competition parameter $p$, from Monte Carlo simulations, for two selected temperatures, as indicated in the figure. The letters $\mathrm{F}, \mathrm{AF}$ and $\mathrm{P}$, denote the ferromagnetic, antiferromagnetic and paramagnetic phases, respectively. The lines joining the squares and the circles represent continuous transition lines.
This work was partially supported by the Brazilian agencies CAPES, CNPq and FUNCITEC(SC).

\section{References}

[1] T. Kaneyoshi, Solid State Communc. 70, 975 (1989); Physica A 205, 677 (1994).

[2] A. L. de Lima, B. D. Stošoć, and I. F. Fittipaldi, J. Magn. Magn. Mater. 226, 635 (2001).

[3] J. A. Plascak, W. Figueiredo, and B. C. S. Grandi, Braz. J. Phys. 29, 579 (1999).

[4] H. F. V. de Resende, F. C. Sá Barreto, and J. A. Plascak, Physica A 149, 606 (1988).

[5] T. Iwashita and N. Uryu, J. Phys. Soc. Jpn. 53, 721 (1984).

[6] G. M. Buendia, M. A. Novotny, and J. Zhang, in: D. P. Landau, K. K. Mon and H. B. Schttler (EDS.), Springer Proceeds in Physics, Vol., 78, Computer Simulations in Condensed Matter Physics VII, Springer, Heidelberg, 1994, p. 223.

[7] G. M. Buendia and M. A. Novotny, J. Phys.: Condens. Matter. 9, 5951 (1997).

[8] J. Marro and R. Dickman, Nonequilibrium Phase Transitions in Lattice Models (Cambridge University Press, Cambridge, 1999).

[9] V. Privman, ed., Nonequilibrium Statistical Mechanics in One Dimension (Cambridge University Press, Cambridge, 1996).

[10] R. J. Glauber, J. Math. Phys. 4, 294 (1963).

[11] M. Godoy and W. Figueiredo, Phys. Rev. E 61, 218 (2000). 